\title{
Circuits neuronaux et comportement. Analyse génétique de traitement olfactif et fonction
}

\author{
(CIRB)
}

\section{Alexander Fleischmann}

\section{OpenEdition}

\section{Journals}

\section{Édition électronique}

URL : https://journals.openedition.org/annuaire-cdf/1088

DOI : 10.4000/annuaire-cdf. 1088

ISBN : 978-2-7226-0325-7

ISSN : 2109-9227

\section{Éditeur}

Collège de France

\section{Édition imprimée}

Date de publication : 1 avril 2013

Pagination : 894-895

ISBN : 978-2-7226-0198-7

ISSN : 0069-5580

\section{Référence électronique}

Alexander Fleischmann, «Circuits neuronaux et comportement. Analyse génétique de traitement olfactif et fonction », L'annuaire du Collège de France [En ligne], 112 | 2013, mis en ligne le 22 novembre 2013, consulté le 22 août 2022. URL : http://journals.openedition.org/annuaire-cdf/1088 ; DOI : https://doi.org/10.4000/annuaire-cdf.1088 
incluant vraisemblablement des agrégats à la surface de la bactérie. Ces résultats ont également été vérifiés par une approche biochimique lors de tests de translocation. Finalement, nous avons observé que contrairement à la souche sauvage d'EPEC, la souche mutante $\operatorname{espC}$ induisait une incorporation importante de marqueur fluorescent dépendant du T3SS lors du contact avec la cellule épithéliale, un test que nous avons mis au point pour évaluer la formation du pore associé au translocon du T3SS. Les résultats obtenus démontrent clairement que l'activité protéolytique d'EspC sur EspD contrôle bien la formation du pore membranaire.

\title{
PUBLICATIONS 2011-2012
}

Tran Van Nhieu G., Liu B.K., Zhang J., Pierre F., Prigent S., Sansonetti P., Erneux C., Kim J.K., Suh P.-G., Dupont G., Combettes L., «Actin-based confinement of calcium responses during Shigella invasion », Nature Communications, sous presse.

Romero S., Quatela A, Bornschlögl T., Guadagnini S., Bassereau P. et Tran Van Nhieu G. « Filopodium retraction is controlled by adhesion to its tip », J. Cell Sci., 16, 2012.

Ehsani S., Santos J.C., Rodrigues C.D., Henriques R., Audry L., Zimmer C., Sansonetti P., Tran Van Nhieu G. et Enninga J., « Hierarchies of Host Factor Dynamics at the Entry Site of Shigella flexneri during Host Cell Invasion », Infect. Immun., 80(7), 2012, 2548-2557; Epub 23 avril 2012.

Romero S., Grompone G., Carayol N., Mounier J., Guadagnini S., Prevost M.C., Sansonetti P.J. et Tran Van Nhieu G., « ATP-Mediated Erk1/2 Activation Stimulates Bacterial Capture by Filopodia, which Precedes Shigella Invasion of Epithelial Cells », Cell Host \& Microbe, 9(6), 2011, 508-519.

Park H., Valencia-Gallardo C., Sharff A., Tran Van Nhieu G. et Izard T., « Novel Vinculin Binding Site of the IpaA Invasin of Shigella. », J. Biol. Chem., 286(26), 2011, 23214-23221.

\section{Circuits neuronaux et comportement. Analyse génétique de traitement olfactif et fonction (CIRB)}

\author{
Responsable : Alexander FLEISCHMANN
}

\section{RECHERCHE}

Notre laboratoire s'intéresse aux circuits neuronaux qui sous-tendent la perception des odeurs. Nous utilisons une combinaison d'approches expérimentales chez la souris, incluant génétique moléculaire, imagerie in vivo et analyse du comportement, pour comprendre la logique du codage de l'information sensorielle par les différents relais olfactifs du cerveau.

La perception des odeurs repose d'une part sur la reconnaissance des divers composants odorifères en périphérie, et d'autre part sur des mécanismes généraux dans le cerveau qui permettent la discrimination des odeurs. Chez les souris, les 
odeurs sont reconnues par des récepteurs olfactifs exprimés à la surface des neurones sensoriels de l'épithélium olfactif. Chaque odeur active un sous-ensemble de neurones sensoriels, et est représentée par un pattern d'activité glomérulaire spatialement invariant dans le bulbe olfactif, le premier centre de traitement de l'information olfactive.

L'information encodée par l'activité des glomérules est ensuite transmise par les cellules mitrales et moufetées vers divers centres olfactifs de plus haut-niveau, qui pourraient être impliquées dans la production des réponses comportementales à partir des représentations corticales.

\section{Des souris au «nez monoclonal » : un indice du traitement de l'information sensorielle}

Afin d'étudier les propriétés fonctionnelles des circuits impliqués dans le traitement de l'information olfactive, nous avons perturbé les patterns d'activité neuronale induits par les stimuli olfactifs en développant une souris transgénique dont $95 \%$ des neurones sensoriels expriment le récepteur olfactif à l'acétophénone, nommé M71.

Des expériences d'imagerie biphotonique in vivo et des analyses comportementales de ces souris nous ont suggéré un modèle du traitement de l'information olfactive selon lequel la reconnaissance de motifs d'activité neuronale au dessus du bruit de fond (phénomène de contraste) est cruciale pour la détection des odeurs.

Pour mettre à l'épreuve ce modèle, nous employons des techniques de pointe d'imagerie in vivo pour comprendre comment le cerveau génère les représentations corticales d'odeurs à partir des patterns d'activité glomérulaire. Nous utilisons des lignées de souris génétiquement modifiées pour caractériser l'activité neuronale induite par les odeurs dans les différents types de neurones du cortex olfactif. Enfin, nous utilisons des approches optogénétique pour analyser l'organisation fonctionnelle de ces différents circuits neuronaux et leurs rôles dans les réponses comportementales aux odeurs.

Le laboratoire utilise une approche interdisciplinaire, combinant génétique moléculaire de la souris, imagerie fonctionnelle in vivo et paradigmes comportementaux pour étudier ces questions clés de biologie sensorielle et neurosciences.

\section{PUBLICATIONS 2011-2012}

Angelo K., Rancz E.A., Pimentel D., Hundahl C., Hannibal J., Fleischmann A., Pichler B., Margrie T.W., «A biophysical signature of network affiliation and sensory processing in mitral cells », Nature, 488(7411), 2012, 375-8.

Glinka M.E., Samuels B.A., Diodato A., Teillon J., Feng Mei D., Shykind B.M., Hen R., Fleischmann A., "Olfactory deficits cause anxiety-like behaviors in mice », J. Neurosci., 32(19), 2012, 6718-25.

Choi G.B., Stettler D.D., Kallman B.R., Bhaskar S.T., Fleischmann A., Axel R., « Driving opposing behaviors with ensembles of piriform neurons », Cell., 146(6), 2011, 1004-15. 